\title{
High-order discontinuous Galerkin methods for computational electromagnetics and uncertainty quantification
}

\author{
J. S. Hesthaven ${ }^{1}$, T. Warburton ${ }^{2}$, C. Chauviere ${ }^{3}$, and L. Wilcox ${ }^{4}$
}

\begin{abstract}
We discuss the basics of discontinuous Galerkin methods (DG) for CEM as an alternative of emerging importance to the widely used FDTD. The benefits of DG methods include geometric flexibility, high-order accuracy, explicit timeadvancement, and very high parallel performance for large scale applications. The performance of the scheme shall be illustrated by several examples. As an example of particular interest, we further explore efficient probabilistic ways of dealing with uncertainty and uncertainty quantification in electromagnetics applications. Whereas the discussion often draws on scattering applications, the techniques are applicable to general problems in CEM.
\end{abstract}

\section{Introduction}

The simplicity, robustness, and reasonable accuracy of the classical finite-difference time-domain (FDTD) method [14] for solving the time-domain Maxwell's equations has propelled this method to become the method of choice among engineers and scientist solving Maxwell's equations in the time-domain. The last decade has seen an explosion in applications and developments, many driven by the very influential texts by Taflove $[11,12]$.

By now it is also clear, however, that the FDTD methods have severe limitations, e.g., its inherent 2 nd order accuracy severely limits its ability to correctly represent wave motion over long distances unless the grid is prohibitively fine. Furthermore,

\footnotetext{
Division of Applied Mathematics, Brown University, Providence, RI 02912, USA Jan. Hesthaven@Brown.edu . Department of Computational and Applied Mathematics, Rice University, Houston, TX 77005, USA timwar@rice.edu . Laboratoire de Mathématiques, Université Blaise Pascal, 63177 Aubiè re, France cedric.chauviere@math.univ-bpclermont.fr. Institute for Computational Engineering and Sciences (ICES), University of Texas at Austin, Austin, TX 78712, USA lucaswlices. utexas. edu
} 
the simplicity of the method, on one hand its very strength, also becomes its most severe restriction by prohibiting the accurate representation of problems in complex geometries.

For the accurate and efficient modeling of large scale EM applications the shortcomings of low order methods render them impractical due to the need for fine grids to avoid prohibitive error accumulation. However, this understanding of the very source of the limitations also suggest that a high-order time-domain solution technique may offer the efficiency and accuracy required for future large scale CEM modeling capabilities. High-order methods are characterized by being able to accurately represent wave propagation over very long distances, using only a few points per wavelength and with an error accumulation rate that is significantly reduced as compared to 2nd order accurate schemes [9]. For three-dimensional applications, this translates into dramatic reductions in the required computational resources, i.e., memory and execution time, and promises to offer the ability to model problems of a realistic complexity and size.

In the following we discuss some of the basic elements of discontinuous Galerkin methods with an emphasis on time-domain electromagnetics. As we will see, these recent developments have paved the way for overcoming many of the restrictions associated with classical high-order methods. In contrast to high-order schemes based on classical finite element techniques, the approach taken here leads to fully explicit schemes.

\section{The Discontinuous Galerkin Method}

The time-dependent Maxwell's equations in the scattered field formulation are given as

$$
\begin{aligned}
\varepsilon \frac{\partial \mathbf{E}^{s}}{\partial t} & =\nabla \times \mathbf{H}^{s}+\sigma \mathbf{E}^{s}+\mathbf{S}^{E}, \\
\mu \frac{\partial \mathbf{H}^{s}}{\partial t} & =-\nabla \times \mathbf{E}^{s}+\mathbf{S}^{H},
\end{aligned}
$$

where, $\mathbf{E}^{s}$ and $\mathbf{H}^{s}$ denote the scattered electric and magnetic fields, $\varepsilon(\mathbf{x})$ and $\mu(\mathbf{x})$ are the local permittivity and permeability, $\sigma(\mathbf{x})$ is the conductivity of the media and $\mathbf{S}^{E}$ and $\mathbf{S}^{H}$ are source terms. Here we have not explicitly written the divergence constraints assuming that the initial conditions satisfy these constraints. Taking the divergence of equations (1)-(2) verifies that if the initial conditions satisfy the divergence constraints then the solution to Maxwell's equations (1)-(2) will also satisfy the divergence constraints.

Let the incident field $\left(\mathbf{E}^{i}, \mathbf{H}^{i}\right)$ be a solution to Maxwell's equations in a media with permittivity, permeability, and conductivity $-\varepsilon^{i}(\mathbf{x}), \mu^{i}(\mathbf{x}), \sigma^{i}(\mathbf{x})$, respectively. Along a perfect electric conductor (PEC), the boundary conditions on the total electric field $\mathbf{E}^{t}=\mathbf{E}^{i}+\mathbf{E}^{s}$ and the total magnetic field $\mathbf{H}^{t}=\mathbf{H}^{i}+\mathbf{H}^{s}$ are 


$$
\hat{\mathbf{n}} \times \mathbf{E}^{t}=\mathbf{0}, \quad \mathbf{H}^{t} \cdot \hat{\mathbf{n}}=0,
$$

where $\hat{\mathbf{n}}$ is the outward pointing normal vector at the surface.

We now briefly describe the computational methods used for solving Maxwell's equations (1)-(2) in the physical space. A discontinuous Galerkin method is used as this offers a number of advantages over widely used alternatives (see [8] for a thorough discussion) and we shall simply sketch its main components. First, we rewrite Maxwell's equations (1)-(2) in conservation form

$$
\mathbf{Q} \frac{\partial \mathbf{q}}{\partial t}+\nabla \cdot \mathbf{F}(\mathbf{q})=\mathbf{S},
$$

where

$$
\mathbf{q}=\left(\begin{array}{c}
\mathbf{E} \\
\mathbf{H}
\end{array}\right), \quad \mathbf{F}_{i}(\mathbf{q})=\left(\begin{array}{c}
-\mathbf{e}_{i} \times \mathbf{H} \\
\mathbf{e}_{i} \times \mathbf{E}
\end{array}\right),
$$

signify the state vector $\mathbf{q}$ and the components of the tensor $\mathbf{F}$ and $\mathbf{e}_{i}$ denotes the Cartesian unit vectors. On the right-hand side of (4), $\mathbf{S}=\left[\mathbf{S}^{E}, \mathbf{S}^{H}\right]$ is the source term, which depends on the incident field, and the material matrix $\mathbf{Q}$ is a diagonal matrix with values $(\varepsilon, \varepsilon, \varepsilon, \mu, \mu, \mu)$ on its diagonal. We assume that the computational domain, $\Omega$, is tessellated by triangles in two spatial dimensions and tetrahedrons in three spatial dimensions, similar to what is done in a finite element/finite volume method.

Given an element $D$ of the tessellation, we represent the local solution $\mathbf{q}_{N}$ restricted to $D$ is given as

$$
\mathbf{q}_{N}(\mathbf{x}, t)=\sum_{i=1}^{N} \widetilde{\mathbf{q}}_{i}(t) L_{i}(\mathbf{x}),
$$

where $\widetilde{\mathbf{q}}_{i}$ reflects nodal values, defined on the element. The function $L_{i}(\mathbf{x})$ signifies an $n$th order Lagrange polynomial $(N=(n+1)(n+2) / 2$ for triangles and $N=(n+$ 1) $(n+2)(n+3) / 6$ for tetrahedrons), associated with grid points on the reference element as illustrated in Figure 1 (see [8] for details).

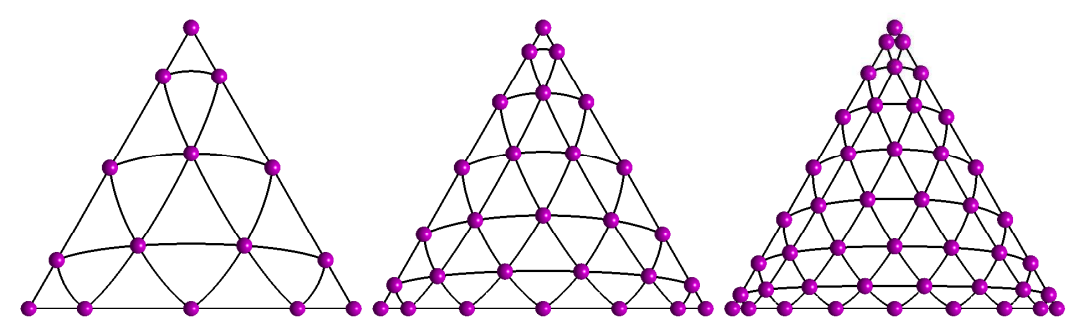

Fig. 1 Examples of nodal sets on the equilateral triangle for orders 4, 6, and 8 .

The discrete solution, $\mathbf{q}_{N}$, of Maxwell's equations is required to satisfy 


$$
\begin{aligned}
& \int_{D}\left(\mathbf{Q} \frac{\partial \mathbf{q}_{N}}{\partial t}+\nabla \cdot \mathbf{F}\left(\mathbf{q}_{N}\right)-\mathbf{S}_{N}\right) L_{i}(\mathbf{x}) d \mathbf{x} \\
= & \oint_{\partial D} \hat{\mathbf{n}} \cdot\left[\mathbf{F}\left(\mathbf{q}_{N}\right)-\mathbf{F}^{*}\right] L_{i}(\mathbf{x}) d \mathbf{x} .
\end{aligned}
$$

In (7), $\mathbf{F}^{*}$ denotes a numerical flux, the expression of which is given as

$$
-\left[\hat{\mathbf{n}} \times \mathbf{H}-(\hat{\mathbf{n}} \times \mathbf{H})^{*}\right]=-\frac{1}{2\{\{Z\}\}} \hat{\mathbf{n}} \times\left[Z^{+}\left(\mathbf{H}^{-}-\mathbf{H}^{+}\right)-\alpha \hat{\mathbf{n}} \times\left(\mathbf{E}^{-}-\mathbf{E}^{+}\right)\right],
$$

and

$$
\left[\hat{\mathbf{n}} \times \mathbf{E}-(\hat{\mathbf{n}} \times \mathbf{E})^{*}\right]=\frac{1}{2\{\{Y\}\}} \hat{\mathbf{n}} \times\left[Y^{+}\left(\mathbf{E}^{-}-\mathbf{E}^{+}\right)+\alpha \hat{\mathbf{n}} \times\left(\mathbf{H}^{-}-\mathbf{H}^{+}\right)\right],
$$

for the equations for the electric and magnetic fields, respectively. Here $\hat{\mathbf{n}}$ is an outward pointing unit vector defined at the boundary $\partial D$ of the element $D$. Using standard notation, $\{\{A\}\}$ signify the average across the interface.

In both cases, we have the possibility of the piecewise constant material coefficients, represented by

$$
Z^{ \pm}=\frac{1}{Y^{ \pm}}=\sqrt{\frac{\mu^{ \pm}}{\varepsilon^{ \pm}}}
$$

as the local impedance and conductance, respectively. The parameter $\alpha$ is a free parameter with $0 \leq \alpha \leq 1$. For $\alpha=0$ the scheme is energy conserving but has a potential for nonphysical solutions in rare cases [8]. For $\alpha>0$, the scheme is slightly dissipative.

Note that this is an entirely local formulation where the fluxes are responsible for coupling of the elements and interchange of information to ensure that the union of the local solutions provides the global solution. Relaxing the continuity of the elements decouples the elements, resulting in a block-diagonal global mass matrix which can be trivially inverted in preprocessing. After discretization of the operators and evaluation of the integrals appearing in (7), the problem can be rewritten in matrix-vector form (see [8])

$$
\mathbf{Q M} \frac{d \mathbf{q}_{N}}{d t}+\mathbf{S} \cdot \mathbf{F}_{N}-M \mathbf{S}_{N}=\mathbf{F} \hat{\mathbf{n}} \cdot\left[\mathbf{F}_{N}-\mathbf{F}^{*}\right] .
$$

The matrices $\mathbf{M}, \mathbf{S}$, and $\mathbf{F}$ represent the local mass-, stiffness-, and face-integration matrices, respectively, the exact entries of which only depend on the metric of the element. The local nature of the scheme allows for the use of an explicit solver for the time discretization of (8) and this is done using an explicit fourth-order RungeKutta method. Purely local time advancements are also possible.

The analysis of the scheme given above is complete and one can prove both stability and high-order accuracy is the solution is smooth enough [8]. In particular, for the dissipative upwind flux $\alpha=1$, one can generally expect optimal accuracy of like $\left\|\mathbf{q}-\mathbf{q}_{N}\right\| \leq C h^{n+1}$ for $h$ being a measure of the cell size. 
To illustrate the performance of the scheme, we consider plane wave TM scattering of a $k a=20 \pi$ metallic cylinder. As simple as the case is, it allows for a thorough validation through the exact solution. We use 950 elements and an high-order local boundary condition [4]. A snapshot of $E^{z}$ is shown in Fig. 2.

To measure the accuracy of the solution we compute the error in $E^{z}$ as a function of time for increasing resolution. The results are shown in Fig. 2. For 5th order polynomials $(n=5)$ there are 8-10 points per wavelength. The results confirm exponential convergence as expected. This is also a indication of the excellent performance of the high order local boundary conditions which introduces errors well below the approximation error.
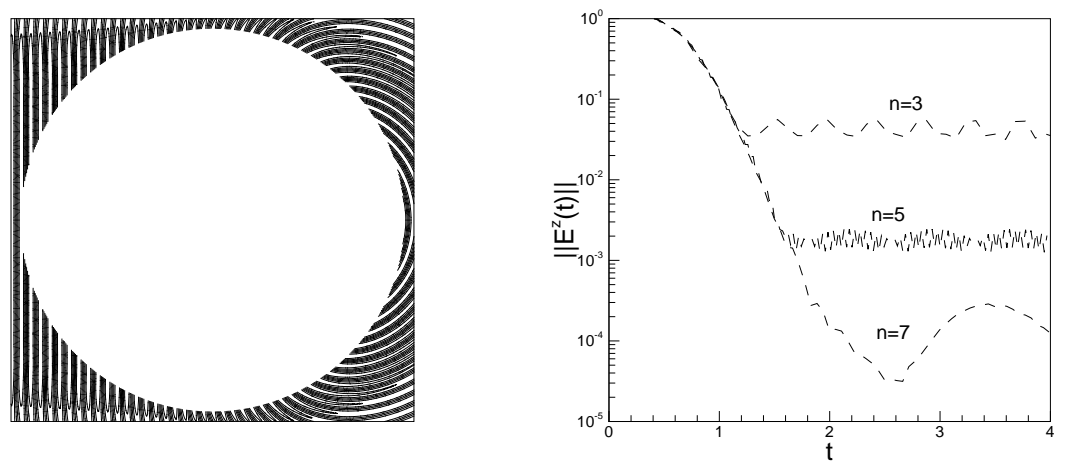

Fig. 2 On the left is shown plane wave TM scattering of a $k a=20 \pi$ metallic cylinder. The snapshot is for $E_{z}$. On the right we show the error in $E^{z}$ for plane wave TM scattering by a $k a=20 \pi$ metallic cylinder as a function of time for increasing resolution.

As a considerably more challenging problem, let us consider scattering by a perfectly conducting business card sized metallic plate as illustrated in Fig. 3. The horizontally polarized plane wave impinges at the metallic plate at an almost grazing angle, causing the excitation of strong waves along the edges of the metallic plate as well as along the length of the plate. These waves contribute significantly to the scattering process and need to be resolved to accurately predict the far field scattering. In Fig. 3 we also show the comparison between the experimentally measured monostatic RCS [13] and a number of particular computed data points. We observe good agreement over the full azimuthal range with results well within the experimental error. We note in particular the good agreement in the backscatter region where the scattering is dominated by traveling waves.

Many further examples and validation tests can be found in [5-7]. 
a)

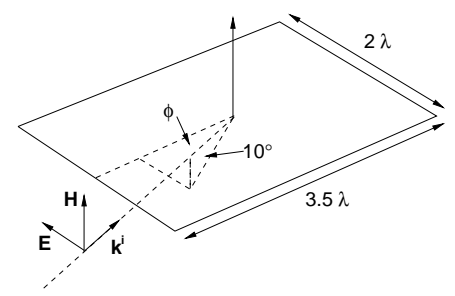

b)

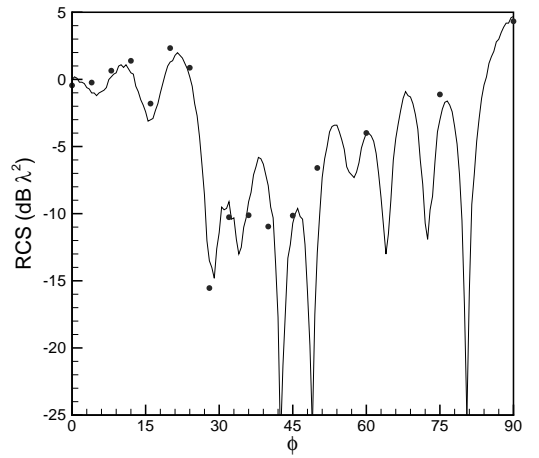

Fig. 3 In a) we show the geometry for the plane wave scattering by a metallic business card while b) shows the comparison between monostatic RCS experimental results [13] (full line) for horizontal polarization of the illuminating field and particular computed data points $(\cdot)$.

\section{Modeling Uncertainty in CEM}

While computational methods have become increasingly refined and accurate, their reliance on exact data, e.g., complete descriptions of geometries, materials, sources etc, are emerging as a bottleneck in the modeling of problems of realistic complexity. For instance, if one attempts to model an experiment, a classic computational approach requires knowledge to a degree of detail which is unrealistic and often impossible to obtain, e.g., one can not hope to control all elements of an experiment, measure all details of an initial condition or geometry, know the microstructure of all materials etc.

The usual approach to deal with this lack of knowledge or uncertainty is to simply assume some mean parameters and compute the corresponding solution. If the solution is robust to parameter variation, this is indeed a reasonable approach. However, for general problems where the sensitivity of parts of the solution can be significant, a solution based on mean parameters is not likely to match very well with experiments and, thus, is not a good predictive tool. We would like to be able to model the impact of the uncertainty, assumed to have certain properties derived from experiments or otherwise, on the computed results, essentially resulting in an ensemble of possible solution values with an associated probabilities which would immediately enable the computation of statistical moments, e.g., means and variances.

As an advanced application of the computational framework presented above, let us here pursue this goal and present a systematic, accurate, and efficient way of addressing this type of problem, built on top of high-order accurate discontinuous Galerkin methods for solving the time-domain Maxwells equations.

The key result on which we shall rely is due to Wiener (1938) (see also Cameron and Martin [1] ) and shows that the Chaos expansion can be used to approximate 
any functional in $L^{2}(\Omega, \mathscr{P})$ where $\mathscr{P}$ is a Gaussian measure on $\Omega$. For such random processes $X(\theta)$, the Chaos expansion reads

$$
X(\theta)=a_{0} H_{0}+\sum_{i_{1}=1}^{d} a_{i_{1}} H_{1}\left(\xi_{i_{1}}(\theta)\right)+\sum_{i_{1}=1}^{d} \sum_{i_{2}=1}^{i_{1}} a_{i_{1} i_{2}} H_{2}\left(\xi_{i_{1}}(\theta), \xi_{i_{2}}(\theta)\right)+\ldots,
$$

where $\xi=\left(\xi_{1}(\theta), \ldots, \xi_{d}(\theta)\right)$ represents $d$ independent Gaussian variables with zero mean and unit variance, each depending on the random event $\theta$, and $H_{n}$ are the multivariate Hermite polynomials. Clearly the number of terms in the expansion (9) grows as

$$
P=\frac{(n+d) !}{n ! d !},
$$

where $n$ is the length of the Hermite expansion and $d$ is the dimension of the Gaussian random space. To model the impact of uncertainty on the propagation of electromagnetic waves, we include the randomness in the usual spatial-temporal dimensions, i.e., the electric field and the magnetic field become $\mathbf{E}(\mathbf{x}, t, \theta)$ and $\mathbf{H}(\mathbf{x}, t, \theta)$, reflecting that the fields are functions of $d$ independent random variables, $\left(\xi_{i_{1}}(\theta), \ldots, \xi_{i_{d}}(\theta)\right)$.

In the following we shall discuss in some detail how this can be utilized to construct an efficient computational method. For simplicity of the discussion, we assume in the sequel that one Gaussian variable suffices to represent the process (i.e. $d=1$ ). However, the formulation is general and applies to problems which require many random variables to describe the stochastic processes.

Using the Chaos expansion we can express $\mathbf{q}(\mathbf{x}, t, \theta)=(\mathbf{E}(\mathbf{x}, t, \theta), \mathbf{H}(\mathbf{x}, t, \theta))^{T}$ as

$$
\mathbf{q}(\mathbf{x}, t, \theta)=\sum_{i=1}^{P} \mathbf{q}^{i}(\mathbf{x}, t) \Psi_{i}(\theta) .
$$

We can write the computational scheme, taking into account the randomness in a general setting, as

$$
\left\{\begin{array}{l}
Q(\theta) M \frac{d \mathbf{q}_{N}}{d t}+S \cdot \mathbf{F}_{N}-M \mathbf{S}(\theta)_{N}=F \hat{\mathbf{n}} \cdot\left[\mathbf{F}_{N}-\mathbf{F}^{*}\right] \\
\mathbf{q}_{N}(\mathbf{x}, t=0, \theta)=\mathbf{f}(\mathbf{x}, \boldsymbol{\theta})
\end{array}\right.
$$

where the initial conditions are given by the function $\mathbf{f}=\mathbf{f}(\mathbf{x}, \theta)$ and the unknown vector $\mathbf{q}_{N}$ is given by (11). As a first step, we discretize (12) in the random space using a Galerkin approach. Multiplying (12) by a test function $\Psi_{k}(\theta)$, replacing $\mathbf{q}_{N}$ by its Chaos expansion and using orthogonality under the Gaussian measure, we obtain

$$
\forall k \in[1, P]: \sum_{i=1}^{P}\left\langle Q \Psi_{i}, \Psi_{k}\right\rangle M \frac{d \mathbf{q}_{N}^{i}}{d t}+k ! S \cdot \mathbf{F}_{N}^{k}-M \mathbf{S}_{N}^{k}=F \sum_{i=1}^{P} \hat{\mathbf{n}} \cdot\left[\mathbf{F}_{N}^{i}-\mathbf{F}^{i *}\right] .
$$

The initial conditions in (12) also need to be projected on to the Chaos basis to give an initial condition for each mode of $\mathbf{q}_{N}^{i}$ in the Chaos expansion, i.e. 


$$
\forall i \in[1, P]: \mathbf{q}_{N}^{i}(\mathbf{x}, t=0)=\frac{1}{i !}\left\langle\mathbf{f}(\mathbf{x}, \theta), \Psi_{i}\right\rangle
$$

Considering Eq.(13) we observe that we have recast the general stochastic problem into a system of $P$ coupled deterministic problems which we can now discretize in space/time as discussed in Sec. 2.
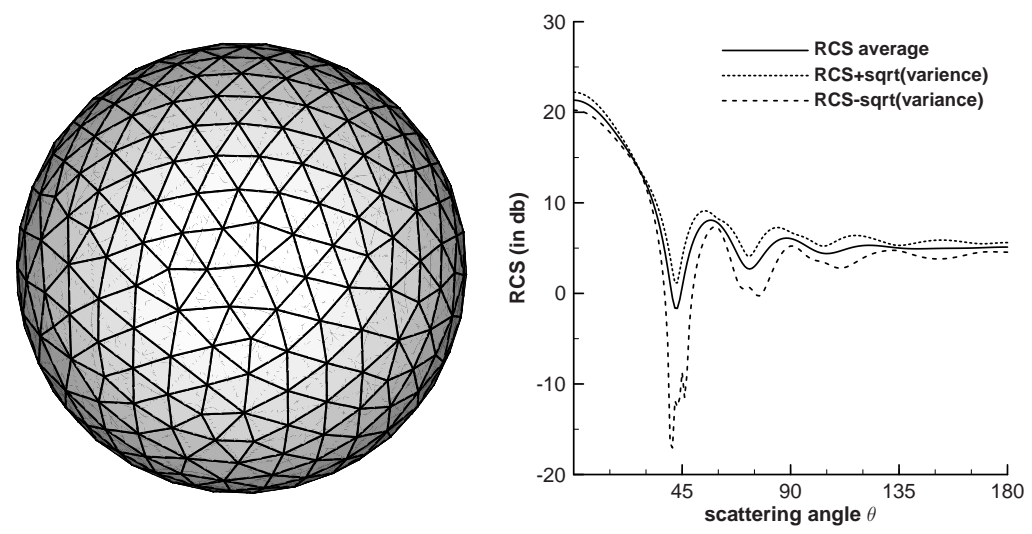

Fig. 4 On the left we show one sample of a surface mesh for the sphere with a random radius and the right illustrates the RCS with uncertainty in the radius of the sphere.

Once the vectors $\left\{\mathbf{q}_{N}^{i}\right\}_{1 \leq i \leq P}$ of the system (13) have been computed, we have available at every point in space an approximation to the probability density of the solution of the system. If we assume that we seek the moments of the solutions or a linear combination of them we can take advantage of the basis to obtain

$$
\langle\mathbf{q}(\mathbf{x}, t, \theta), 1\rangle=\sum_{i=1}^{P} \mathbf{q}^{i}(\mathbf{x}, t) \delta_{1 i}=\mathbf{q}^{1}(\mathbf{x}, t),
$$

i.e., the average is simply the first mode in the Chaos expansion. In a similar way, we can obtain the variance and higher moments. Often, however, we are interested in the statistics of some derived, possibly non-linear, functional, $F(\mathbf{q})$ of $\mathbf{q}(\mathbf{x}, t, \theta)$, e.g., computation of the impact on the radar cross section (RCS) of the uncertainty in the scattering problem. To achieve this we consider

$$
F(\mathbf{q}(\theta))=\sum_{j=1}^{P} F\left(\mathbf{q}\left(\theta_{j}\right)\right) L_{j}(\theta)
$$

i.e.,, we simply need to evaluate the general functionals at the values of $\theta_{j}$ and since we have already obtained full probabilistic information in the expansions we can use these results directly to obtain the required information and, thus, the probabilistic information on $F(\mathbf{q})$. All informations of interest, e.g., moments, can now 
be extracted from this in the same way as for the simple variables. Naturally, one can evaluate the integrals using a classic Monte Carlo approach. This can be done at little cost since it only requires evaluation of the expansions and not solution of Maxwell's equations.

For the first experiment we consider the scattering of a plane wave, with normalized frequency $\omega=1$, from a PEC sphere. We assume the sphere has a uniformly distributed random radius in the interval $[0.9 \lambda, 1.1 \lambda]$, where $\lambda$ is the wavelength of the incident field. For the spatial discretization we use fourth order elements and a sample mesh is presented in Figure 4(restricted to the surface of the sphere) and we show the average of the RCS and the possible variations around its average value.
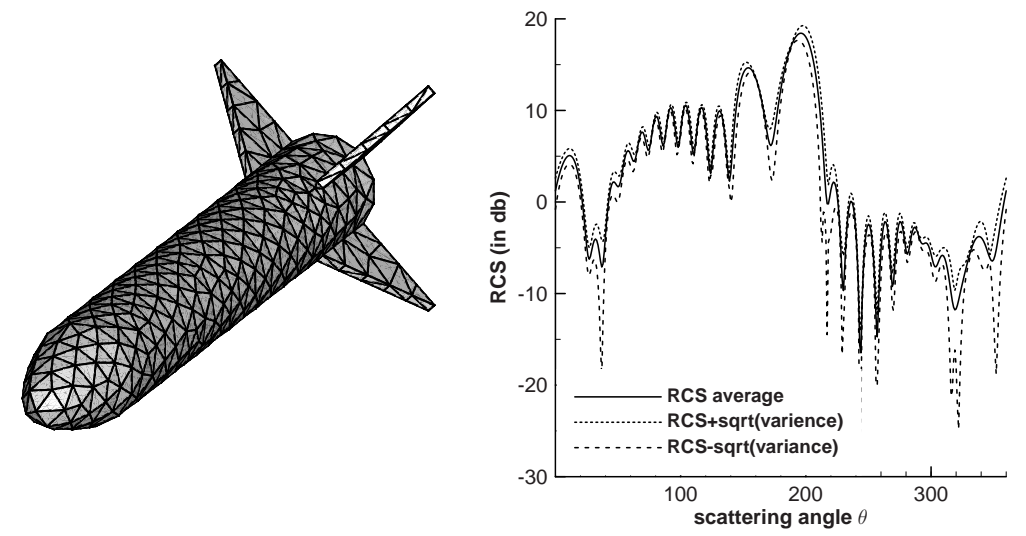

Fig. 5 On the left we show the surface mesh for the three-dimensional rocket and on the right the RCS for the three-dimension rocket problem. Results are shown with the mean RCS as well as \pm one standard deviation.

For the second example we consider the scattering of a plane wave, with frequency $\omega=1$, from a PEC rocket. The direction of the incident field is assumed to be unknown but uniformly distributed in the interval $[10,20]$ degrees. For this calculation the physical space is discretized with degree five polynomials in each element. Figure 5 shows the mesh (restricted to the surface of the rocket) and the average of the RCS and the possible variations around its average value.

\section{Final Remarks}

The discontinuous Galerkin method is at this stage a robust, efficient, accurate and thoroughly validated alternative to the more classic FDTD method. It overcomes many of the problems with both FDTD methods and alternatives such as finite- 
volume and finite element methods. Furthermore, large scale software [10] is available for download and use and there are several examples of successful third party use. In this paper we have focused on PEC objects but there is nothing special about these. The method is entirely general and can accomodate general materials, including anisotropic and nonlinear materials as needed. Furthermore, the efficiency of the method has been demonstrated on large problems already.

We have also discussed the combination of these techniques with more recent developments to enable the modeling of PEC objects with random shapes and uncertainties in the incident field. The approach described can, however, equally well be used to account for others types of uncertainties as well as in connection with other computational techniques. For example, instead of being purely reflective, the object can be a material with a random shape. In this case, it is necessary to mesh the entire domain and define a permittivity $\varepsilon$ that takes some value inside the object and another value outside. For material objects, the shape of the objects can be moved randomly in the same way as a PEC object. In [2,3], the uncertainty in the shape of a material object was studied. However, the approach used was limiting the uncertainty to be modeled to a single random variable. Other types of uncertainties were also studied (randomness of the source term to mimic a slight variation in the frequency of the source, randomness of the permittivity).

The combination of these two methods offers a unique ability to model large scale time-dependent EM problems at high accuracy and with the ability to accurately and efficiently account of sources of uncertainty, leading to sensitivity estimates of measures of interest, e.g., the radar cross section.

Acknowledgements The authors appreciate the partial support of AFOSR under grant FA955004-1-0072 and FA9550-07-1-0425.

\section{References}

1. R. H. Cameron and W. T. Martin, The Orthogonal Development of Nonlinear Functionals in Series of Fourier-Hermite Functionals, Ann. Math. 48 (1947), 385-392.

2. C. Chauvière, J. S. Hesthaven and L. Lurati, Computational modeling of uncertainty in timedomain electromagnetics, SIAM J. Sci. Comput., 28(2006), 751-775

3. C. Chauvière, J. S. Hesthaven and L. Wilcox, Efficient Computation of RCS from Scatters of Uncertain Shapes, IEEE Trans. Antennas Propagat. 55(2007), 1437-1448.

4. T. Hagstrom and T. Warburton, A New Auxiliary Variable Formulation of High-Order Local Radiation Boundary Conditions: Corner Compatibility Conditions and Extensions to First Order Systems, Wave Motion, 2007 - to appear.

5. J. S. Hesthaven and T. Warburton, High-order nodal methods on unstructured grids. I. Timedomain solution of Maxwell's equations, J. Comput. Phys., 181 (2002), 1-34.

6. J. S. Hesthaven and T. Warburton, Discontinuous Galerkin methods for the time-domain Maxwell's equations: An introduction, ACES Newsletter, 19(2004), 10-29.

7. J. S. Hesthaven and T. Warburton, High Order Nodal Discontinuous Galerkin Methods for the Maxwell Eigenvalue Problem, Royal Soc. London Ser A 362(2004), 493-524. 
8. J.S. Hesthaven and T. Warburton, 2008, Nodal Discontinuous Galerkin Methods: Algorithms, Analysis, and Applications. Springer Texts in Applied Mathematics 54, Springer Verlag, New York.

9. H. O. Kreiss and J. Oliger, Comparison of Accurate Methods for the Integration of Hyperbolic Problems, Tellus 24(1972), pp. 199-215.

10. nudg++: www.nudg.org

11. A. Taflove, Computational Electrodynamics. The Finite-Difference Time-Domain Method. Artech House, Boston, 1995.

12. A. Taflove (Ed.), Advances in Computational Electrodynamics: The Finite-Difference TimeDomain Method, Aztech House, Boston, 1998.

13. J. L. Volakis, Benchmark Plate Radar Targets for the Validation of Computational Electromagnetics Programs, IEEE Antennas Propagat. Mag. 34(1992), pp. 52-56.

14. K. S. Yee, Numerical Solution of Initial Boundary Value Problems involving Maxwells Equations in Isotropic Media, IEEE Trans. Antennas Propaga. 14(1966), pp. 302-307. 